Research, Society and Development, v. 9, n. 8, e230985481, 2020

(CC BY 4.0) | ISSN 2525-3409 | DOI: http://dx.doi.org/10.33448/rsd-v9i8.5481

\title{
Elaboração de pão de forma adicionado de yacon
}

Elaboration of yacon bread

Panificación yacon

Recebido: 10/06/2020 | Revisado: 13/06/2020 | Aceito: 19/06/2020 | Publicado: 02/07/2020

Danielly Ramalho de Oliveira

ORCID: https://orcid.org/0000-0002-4215-5735

Centro Universitário Estácio do Ceará, Brasil

E-mail: daniellyramalho@ hotmail.com

Ana Paula Colares de Andrade

ORCID: https://orcid.org/0000-0003-0554-4376

Universidade Federal do Ceará, Brasil

E-mail: ana.colares@ hotmail.com

\section{Resumo}

Yacon (Smallanthus sonchifolius) é uma raiz tuberosa de origem Andina, considerada um alimento funcional, por conter fruto-oligossacarídeos (FOS) e compostos fenólicos. O consumo de FOS melhora o crescimento de bifidobactérias no intestino, o metabolismo gastrointestinal e estimula a absorção do cálcio, tem efeito antioxidante, reduz os níveis de colesterol e de glicose sérico. Através desse trabalho, objetivou-se avaliar o aproveitamento integral da batata yacon na elaboração de pães de forma. Foram elaborados 3 pães com concentrações de $5 \%$, onde a primeira formulação foi composta pela farinha da polpa, a segunda contendo a farinha da casca e a terceira utilizando as farinhas da casca e da polpa da batata. Após o processamento, as formulações foram submetidas às análises microbiológicas onde apresentaram resultados negativos para coliformes totais e fecais e Salmonella sp. Os resultados do teste de ordenação por preferência e da análise sensorial para os atributos textura e sabor demonstraram que não houve diferença significativa entre nenhuma das formulações. A formulação 2 com a farinha da casca e a formulação 1 com a farinha da polpa, apresentaram uma boa aceitação, com relação a intenção de compra, indicando possibilidade de comercialização destes produtos. Os resultados obtidos neste estudo contribuíram de forma significativa para estimular o aproveitamento integral da batata yacon na elaboração de um pão diferenciado, com potencial para a comercialização e podendo apresentar um alto valor nutricional. 
Palavras-chave: Alimento funcional; Fibra alimentar; Produto de panificação.

\begin{abstract}
Yacon (Smallanthus sonchifolius) is a tuberous root of Andean origin, considered a functional food, because it contains fructo-oligosaccharides (FOS), and phenolic compounds. The consumption of FOS improves the growth of bifidobacteria in the intestine, gastrointestinal metabolism and stimulates calcium absorption, has antioxidant effect, reduces cholesterol and serum glucose levels. Through this work, the objective was to evaluate the integral use of yacon potato in the elaboration of form loaves. Three loaves were prepared with concentrations of $5 \%$, where the first formulation was composed of the flour of the pulp, the second containing the flour of the bark and the third using the flour of the bark and the pulp of the potato. After processing, the formulations were submitted to microbiological analyzes where they showed negative results for total and fecal coliforms and Salmonella. The results of the preference sorting test and sensory analysis for the attributes texture and flavor showed that there was no significant difference between any of the formulations. Formulation 2 with the shell flour and formulation 1 with the pulp flour, showed good acceptance in relation to the purchase intention, indicating the possibility of commercialization of these products. The results obtained in this study contributed in a significant way to stimulate the integral use of the yacon potato in the elaboration of a differentiated bread, with potential for commercialization and being able to present a high nutritional value.
\end{abstract}

Keywords: Functional food; Fiber food; Bread product.

\title{
Resumen
}

El yacón (Smallanthus sonchifolius) es una raíz tuberosa de origen andino, considerada un alimento funcional, ya que contiene fructo-oligosacáridos (FOS) y compuestos fenólicos. El consumo de FOS mejora el crecimiento de bifidobacterias en el intestino, el metabolismo gastrointestinal y estimula la absorción de calcio, tiene un efecto antioxidante, reduce los niveles de colesterol y glucosa en suero. A través de este trabajo, el objetivo fue evaluar el uso completo de las papas de yacón en la preparación de hogazas de pan. Se prepararon tres panes con concentraciones de 5\%, donde la primera formulación estaba compuesta por la harina de la pulpa, la segunda contenía la harina de la piel y la tercera usando la harina de la piel y de la pulpa de papa. Después del procesamiento, las formulaciones se sometieron a análisis microbiológicos donde mostraron resultados negativos para coliformes totales y fecales y Salmonella sp. Los resultados de la prueba de orden de preferencia y el análisis 
sensorial para los atributos de textura y sabor demostraron que no había una diferencia significativa entre ninguna de las formulaciones. La formulación 2 con la harina de la cáscara y la formulación 1 con la harina de la pulpa, mostraron una buena aceptación, con respecto a la intención de compra, indicando la posibilidad de comercializar estos productos. Los resultados obtenidos en este estudio contribuyeron significativamente a estimular el uso completo de la papa yacón en la preparación de un pan diferenciado, con potencial de comercialización y poder presentar un alto valor nutricional.

Palabras clave: Alimentos funcionales; Fibra dietética; Producto de panadería.

\section{Introdução}

O estilo de vida e uma dieta inadequada são fatores de risco que vem sendo associado ao desenvolvimento de doenças crônicas não transmissíveis (DCNT), tornando-se motivo de preocupação mundial em função da sua relevância e custo social, gerando elevado número de mortes, perda da qualidade de vida, além de resultado econômico negativo para as famílias (Malta, et al., 2014). Em 2012, 74\% dos óbitos que ocorreram no Brasil (Malta, et al., 2016) e $70 \%$ das mortes mundiais foram relacionados a doenças crônicas não transmissíveis (Malta, et al., 2017).

A alimentação é vista como um dos aspectos mais importantes no controle e tratamento de algumas dessas doenças (Zaparolli, et al., 2013). Diante disso, vem se tornando crescente o interesse pelos alimentos funcionais, que tem como objetivo colaborar para promoção da saúde (Casemiro \& Ramos, 2015).

Alimentos funcionais são definidos como aqueles que ao serem consumidos na dieta, além das funções nutricionais básicas, apresentam benefícios para o funcionamento metabólico e fisiológico do organismo, contribuindo para a saúde, melhorando a qualidade de vida e prevenindo doenças (Basho \& Bin, 2010). Agência Nacional de Vigilância Sanitária (ANVISA) estabeleceu em lista os nutrientes e não nutrientes com alegação de propriedade funcional, dentre eles está o Fruto-oligossacarídeo (FOS) (Brasil, 2009).

O Fruto-oligossacarídeo (FOS) exerce propriedades fisiológicas e funcionais semelhantes às fibras (Rolim, 2008). Sua ingestão provoca um efeito bifidogênico estimulando seletivamente a proliferação de bifidobactérias, que são bactérias probióticas, ou seja, são benéficas e naturalmente encontradas no intestino (Caetano, et al., 2016). O consumo de FOS melhora ainda o metabolismo gastrointestinal (Delgado, et al., 2013), estimula a absorção do cálcio (Lobo, et al., 2007), tem efeito antioxidante (Castro, et al., 2012), reduz os 
níveis de colesterol sérico (Habib, et al., 2011) e de glicose no sangue (Baroni, et al., 2016), além de promover uma melhora do sistema imunológico (Delgado, et al., 2012).

Para ser considerada fonte de FOS a porção do alimento deve fornecer no mínimo 2,5g deste elemento. O Fruto-oligossacarídeo é um componente alimentar natural que pode ser encontrado no alho, cebola, alcachofra, banana, trigo e na yacon (Brasil, 2012; Caetano, et al., 2016). Esta última tem grande potencial como alimento funcional devido ao seu alto conteúdo de fruto-oligossacarídeos e compostos fenólicos (Delgado, et al., 2013).

O yacon é uma raiz tuberosa, de origem Andina, que pertence à família Asteraceae, também chamada de compositae. Tem como nome científico Smallanthus sonchifolius. É uma espécie que se adequa a diferentes regiões climáticas, altitudes e tipos de solos (Santana \& Cardoso, 2008), sendo cultivada em diferentes países como Argentina, Bolívia, República Checa, Equador, Itália, Japão, Coréia, Nova Zelândia, Peru, Estados Unidos e Brasil (Caetano, et al., 2016).

A partir do yacon é possível se obter a farinha, e com ela desenvolver novos produtos, tais como: bolos, apresuntados, doces e pães (Maldonado \& Singh, 2008; Rolim, 2008; Padilha, et al., 2010; Pereira, et al., 2013; Contado, et al., 2015).

Segundo a Resolução RDC n $^{\circ} 263$, de 22 de setembro de 2005, os pães são produtos obtidos de farinha de trigo e/ou outras farinhas, adicionados de líquido, resultantes do processo de fermentação (ou não) e cocção, podendo conter outros ingredientes, desde que os produtos não sejam descaracterizados. Ele pode, ainda, apresentar cobertura, recheio, formato e textura diversos (Brasil, 2005).

O pão tem sido muito utilizado para fins de enriquecimento nutricional, por ser acessível aos consumidores de diferentes classes sociais, além de apresentar aroma, textura e sabor agradável (Oliveira, et al., 2011; Talma, et al., 2011). E com o aumento da conscientização do consumidor para preservar sua saúde, tem crescido o interesse em agregar ao pão ingredientes como as fibras alimentares (Sivam, et al., 2011).

Estudos mostram que adicionar fibras aos pães pode aumentar seu volume, alterar sua cor, flexibilidade e firmeza. Além de considerável melhora do valor nutricional do produto enriquecido, promovendo aumento no teor de fibras dos pães (Rahaie, et al., 2014).

Diante do exposto, este trabalho propôs elaborar um pão de forma acrescido de farinha de yacon, aproveitando todas as partes do tubérculo. 


\section{Metodologia}

O trabalho teve um caráter exploratório, laboratorial, quantitativo, com delineamento transversal e experimental como preconiza Pereira e colaboradores (2018). Executado nos Laboratórios de Microbiologia e de Tecnologia de Alimentos do Centro Universitário Estácio do Ceará, no período de fevereiro a junho 2018, submetido a Plataforma Brasil e aprovado sob o número 026562/2018. Participaram da pesquisa 70 provadores (IAL, 2008) escolhidos de forma aleatória, de ambos os sexos e não treinados, incluindo estudantes e funcionários da referida instituição.

Para a elaboração dos pães foram utilizadas matérias primas adquiridas no comércio local de Fortaleza (CE). Foram elaboradas três formulações de pães, a saber: primeira formulação F1 continha 5\% da farinha da polpa de yacon, a segunda F2 continha 5\% da farinha da casca e formulação F3 continha 5\% da farinha da casca e da polpa da batata.

\section{Obtenção da farinha da casca e da farinha da polpa de Yacon}

Após a obtenção das batatas, elas foram higienizadas com água corrente e sanitizadas em solução de hipoclorito de sódio a 200 ppm por 10 minutos. Logo após, descascadas para separação de casca e polpa sendo está última fatiada em lâminas de 0,3 cm de espessura. Ambas as partes foram imersas em solução de Sulfito de sódio 5\% durante 15 minutos. Em seguida, foram secadas em estufa com circulação de ar a $55^{\circ} \mathrm{C}$, sendo a casca e a polpa desidratada por 24 horas. Após esse procedimento foram trituradas em processador doméstico para a obtenção das farinhas e armazenadas em recipientes de vidro hermeticamente fechados em temperatura ambiente até o momento da produção dos pães (Ribeiro, 2008; Rolim, 2008).

\section{Processamento do pão adicionado de farinha de Yacon}

Para elaboração dos pães foram utilizadas a farinha da casca e a farinha da polpa de yacon com variação nas proporções. A seguir, encontra-se descrito os ingredientes bem como suas devidas proporções em cada formulação (Tabela 1).

Os ingredientes foram pesados e misturados os secos, logo após foram adicionados os ingredientes líquidos. A massa descansou por 30 minutos em ambiente fechado e depois foi sovada. Em seguida, os pães foram levados para fermentar em forma apropriada, untada com óleo e protegidos por saco plástico durante 60 minutos. Os pães foram assados em forno a 
Research, Society and Development, v. 9, n. 8, e230985481, 2020

(CC BY 4.0) | ISSN 2525-3409 | DOI: http://dx.doi.org/10.33448/rsd-v9i8.5481

$200^{\circ} \mathrm{C}$ por aproximadamente 20 minutos, até que dourasse a superfície. Após retirados das formas, foram resfriados em temperatura ambiente por uma hora, em seguida embalados e guardados por volta de 24 horas até a realização das análises microbiológicas (Costa, 2014).

Tabela 1. Ingredientes e formulações para a elaboração do pão adicionado de farinha de yacon.

\begin{tabular}{cccc}
\hline Ingredientes & $\mathbf{F 1}$ & $\mathbf{F ~ 2}$ & $\mathbf{F ~ 3}$ \\
& $\mathbf{\%}$ & $\mathbf{\%}$ & $\mathbf{\%}$ \\
\hline Farinha de trigo sem fermento & $100 \%$ & $100 \%$ & $100 \%$ \\
Farinha da polpa & $5 \%$ & - & $2,5 \%$ \\
Farinha da casca & - & $5 \%$ & $2,5 \%$ \\
Sal & $2 \%$ & $2 \%$ & $2 \%$ \\
Açúcar cristal & $13 \%$ & $13 \%$ & $13 \%$ \\
Fermento biológico & $3 \%$ & $3 \%$ & $3 \%$ \\
Manteiga & $7 \%$ & $7 \%$ & $7 \%$ \\
Leite integral & $50 \%$ & $50 \%$ & $50 \%$ \\
Ovos & $16 \%$ & $16 \%$ & $16 \%$ \\
\hline
\end{tabular}

Fonte: Adaptado de Rolim (2008).

As três formulações dos pães foram submetidas à análise microbiológica antes da análise sensorial. Seguindo a Resolução RDC nº12/01, onde foram realizadas as análises de coliformes à $45^{\circ} \mathrm{C}$ e Salmonella sp nos pães (Brasil, 2001), segundo a metodologia de Silva, et al. (2010).

Depois de feitas as análises e constatado a inocuidade dos produtos, foram convidados 70 participantes para análise sensorial (IAL, 2008), como critérios de exclusão, não fizeram parte da pesquisa os participantes alérgicos a glúten ou a um dos componentes da elaboração. Os participantes realizaram a análise após assinarem o Termo de Consentimento Livre e Esclarecido obedecendo a Resolução 466/12 do Conselho Nacional de Saúde (Brasil, 2012).

Aplicou-se o teste sensorial de ordenação por preferência, onde as três amostras foram apresentadas aos provadores que organizaram em uma ficha de avaliação sua preferência, na qual 1 representava "menos preferida" e 3 "mais preferida". A amostra preferida, teve seus atributos de textura e sabor avaliados utilizando escala hedônica estruturada de nove pontos, na qual 9 representava "gostei extremamente" e 1 "desgostei extremamente" (IAL, 2008). 
Além disso, foi avaliada quanto à intenção de compra através de escala estruturada de cinco pontos, na qual 5 representou "certamente compraria" e 1 "certamente não compraria" (Meilgaard, et al., 2006; IAL, 2008).

As amostras foram apresentadas aos provadores de forma aleatória, à temperatura ambiente, em pratos descartáveis e codificados com número de três dígitos escolhidos de forma aleatória. Os provadores estavam posicionados em cabines individuais e foram devidamente orientados a observar as características do produto e o preenchimento das fichas de respostas.

Os resultados foram organizados e expressos na forma de tabelas e gráficos com auxílio do Microsoft Excel 2013. As respostas para ordenação por preferência foram submetidas a um tratamento estatístico realizada por meio dos testes de Friedman utilizando a tabela de Newell e MacFarlane (IAL, 2008).

\section{Resultados e Discussão}

Não foram detectados coliformes totais à $45^{\circ} \mathrm{C}$ e Salmonella $s p$ nas formulações, desta forma, as amostras foram submetidas às análises sensoriais. A segurança microbiológica de alimentos é de suma relevância para a saúde dos provadores e realização de testes sensoriais.

O perfil dos 70 provadores foi composto por 68,57\% $(n=48)$ de mulheres e $31,43 \%$ $(n=22)$ de homens, com idade entre 18 e 50 anos e nível superior incompleto. Zavareze \& Basso (2015) ao analisarem a aceitabilidade de biscoitos de amaranto e yacon contaram com público de faixa etária semelhante à deste estudo, com idades entre 19 e 50 anos.

O teste de ordenação por preferência, demonstrou que que não houve diferença estatística entre as amostras (Tabela 2). Os resultados obtidos nesse teste, aos níveis de $1 \%$ e $5 \%$ de probabilidade, demonstraram que apesar de a formulação F1 ter sido a mais bem avaliada, não houve diferença significativa entre nenhuma das formulações analisadas. Isso indica que a adição da farinha composta por casca e polpa de yacon a uma concentração de $5 \%$ não interferiu na aceitabilidade do produto, o que sugere que as três formulações, possuem potencial para comercialização. 
(CC BY 4.0) | ISSN 2525-3409 | DOI: http://dx.doi.org/10.33448/rsd-v9i8.5481

Tabela 2. Resultados do teste Friedman para ordenação por preferência do Pão de Forma Adicionado de yacon.

\begin{tabular}{cccc}
\hline Formulações & F1 & F2 & F3 \\
& $\begin{array}{c}\text { (5\% da farinha da } \\
\text { polpa de yacon) }\end{array}$ & $\begin{array}{c}\text { (5\% da farinha da casca } \\
\text { de yacon) }\end{array}$ & $\begin{array}{c}\text { (5\% da farinha da casca e da } \\
\text { polpa de yacon) }\end{array}$ \\
\hline Somatório total & 34 & 18 & 18 \\
Diferença versus F1 & - & $34-18=16^{\mathrm{a}}$ & $34-18=16^{\mathrm{a}}$ \\
Diferença versus F2 & - & - & $18-18=0^{\mathrm{a}}$ \\
Diferença versus F1 & - & - & - \\
\hline \multicolumn{2}{c}{ Resultados com letras iguais na mesma coluna não indicam diferença significativa. }
\end{tabular}
Fonte: Dados da pesquisa (2018).

Os resultados obtidos no teste de ordenação por preferência (Tabela 2) aos níveis de 1\% e 5\% de probabilidade demonstraram que, apesar de a formulação F1 (5\% da farinha da polpa de yacon) ter sido a mais bem avaliada, não houve diferença significativa entre nenhuma das formulações analisadas. Isso indica que a adição da farinha composta por casca e polpa de yacon a uma concentração de 5\% (formulação F3), não interferiu de maneira significativa na aceitabilidade do produto, sendo assim, as três formulações possuem potencial para comercialização.

Cardoso (2017), constatou em biscoitos tipo cookies enriquecidos com farinha de yacon nas concentrações de $12,5 \%, 25 \%$ e $50 \%$, que aqueles que apresentaram concentrações de até $25 \%$ da farinha da polpa foram os mais bem aceitos.

Em outro estudo Rodrigues, et al. (2014), verificaram que a adição de até 7,5\% de farinha da polpa de yacon em biscoitos tipo cookies trouxe resultados positivos em relação à aceitação do produto pelos provadores.

Zavareze \& Basso (2015) testaram a aceitabilidade de biscoitos substituindo $100 \%$ da farinha de trigo por farinha de amaranto e de yacon e verificaram que os biscoitos com a farinha de amaranto foram mais aceitos do que os que continham farinha de yacon.

O resultado de média e desvio padrão para os atributos sensoriais (sabor e textura) pontuados pelos provadores na avaliação sensorial dos pães encontra-se na Tabela 3. Já o Gráfico 1, demonstra os resultados para a intenção de compra. 
Research, Society and Development, v. 9, n. 8, e230985481, 2020

(CC BY 4.0) | ISSN 2525-3409 | DOI: http://dx.doi.org/10.33448/rsd-v9i8.5481

Tabela 3. Resultado da análise sensorial com aplicação da escala hedônica na avaliação das amostras de pães formulados com farinha de yacon.

\begin{tabular}{ccc}
\hline Formulação & Textura & Sabor \\
\hline F1 & $8,09 \pm 1,06$ & $7,79 \pm 1,37$ \\
F2 & $8,0 \pm 1,03$ & $8,33 \pm 0,91$ \\
F3 & $7,83 \pm 0,71$ & $8 \pm 0,97$ \\
\hline
\end{tabular}

Resultados estão expressos em média e desvio padrão.

Fonte: Dados da pesquisa (2018).

Observa-se que não houve diferença significativa em nenhuma das amostras para os aspectos relacionados à textura e sabor; tal resposta pode estar associada a pequena concentração de farinha adicionada ao produto elaborado.

Pasini \& Doneda (2016), elaboraram 5 formulações de pães com reduzido teor de fenilalanina para pacientes com fenilcetonúria, utilizando-se de mandioca (T1), batata baroa (T2), batata doce (T3), batata inglesa (T4) e batata yacon (T5) e constataram que a amostra que continha batata yacon foi bem avaliada no atributo sabor e textura.

Moraes \& Scheid (2011) avaliaram a aceitação por parte de diabéticos dos aspectos sensoriais de pães produzidos com yacon. Os resultados não indicaram diferença significativa entre a formulação padrão e a formulação que levou a batata yacon in natura, no que se referiu ao sabor.

Gráfico 1. Resultado obtido para o Teste de intenção de compra do pão adicionado de yacon.

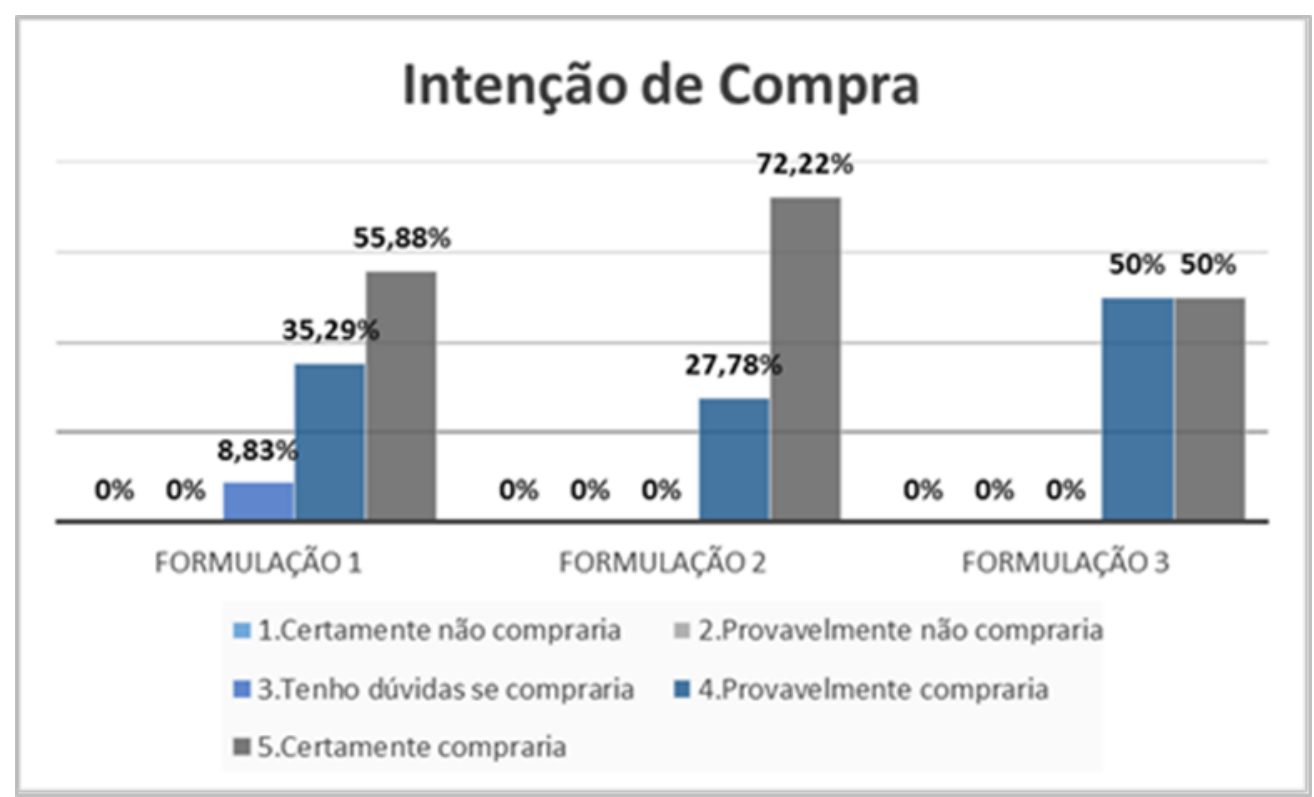

Fonte: Dados da pesquisa (2018). 
Com relação à intenção de compra, os resultados apresentados indicam maior frequência para a formulação F2 que continha farinha da casca, onde, 72,22\% dos participantes certamente compraria o produto. Em segundo lugar ficou a formulação F1 composta pela farinha da polpa, somando $55,88 \%$ das pessoas que certamente compraria. Os testes foram realizados com os mesmos participantes do teste de ordenação por preferência das amostras.

Esse resultado demonstra que a formulação melhor avaliada quanto à intenção de compra entre o público foi a F2, seguida da F1. Isso confirma mais uma vez que ambas possuem relevante potencial comercial, além de ser uma alternativa para a utilização de todas as partes comestíveis da batata.

Goes, et al. (2014) elaboraram cookies com substituição da farinha de trigo pela farinha de resíduos do processamento do extrato da yacon em três formulações com a seguintes concentrações: A1 (padrão com concentração 0\%), A2 (50\%) e A3 (100\%). Após realizarem as análises constataram que apesar de A1 e A2 terem sido as preferidas pelos provadores, não houve diferença estatística entre as três amostras quanto ao sabor. Em relação à intenção de compra verificaram que a formulação com concentração de $50 \%$ da farinha de yacon (A2) foi tão aceita quanto a padrão (A1).

Pesquisas revelam que a yacon pode ser considerada fonte potencial de prébióticos (Cao, et al., 2018), sendo eficaz na melhora dos sintomas da constipação (Sant'anna, et al., 2015). E que, quando ela é adicionada a pães, eles possuem além de potencial prébiótico, índice glicêmico de moderado abaixo, alto teor de fibras e baixo teor de gordura (Rolim, 2011). Pode contribuir também com o aumento da quantidade de elementos minerais ser uma boa opção para celíacos (podendo substituir 100\% da farinha de trigo) (Almeida \& Szlapak, 2015), além de o pão apresentar características sensoriais agradáveis (Moraes \& Scheid, 2011).

Diante do exposto, observou-se que a proposta de elaboração do pão de forma adicionado de yacon (em sua integralidade ou em partes) é viável e que o produto a ser desenvolvido apresenta potencial de consumo e comercialização. Contudo, mais pesquisas serão realizadas para que as características nutricionais do pão possam ser definidas.

\section{Considerações Finais}

Observou-se que não houve diferença significativa entre as amostras avaliadas e que o produto teve uma boa aceitação pelos provadores. Sendo assim, um aproveitamento integral 
da batata yacon pode ser uma alternativa promissora na elaboração de novos produtos, como uma forma de aproveitar o tubérculo, podendo principalmente aumentar o valor nutricional do produto.

Este trabalho continuará sendo executado com o intuito de avaliar a quantidade de fibras, carboidratos, proteínas, lipídios, umidade e cinza presentes nas formulações.

\section{Referências}

Almeida, E. A., \& Szlapak, R. Y. (2015). Elaboração de pão sem glúten adicionado de farinhas de batata yacon, batata doce e batata cará. (Trabalho de Conclusão de Curso). Universidade Tecnológica Federal do Paraná, Curitiba, Paraná.

Baroni, S., Rocha, B. A., Melo, J. O., Comar, J. F., Caparroz-Assef, S. M., \& Bersani-Amado, C. A. (2016). Hydroethanolic extract of Smallanthus sonchifolius leaves improves hyperglycemia of streptozotocin induced neonatal diabetic rats. Asian Pacific Journal of Tropical Medicine, 9(5), 432-6.

Basho, S. M., Bin, M. C. (2010). Propriedades dos alimentos funcionais e seu papel na prevenção e controle da hipertensão e diabetes. Interbio, 4(1), 48-58.

Brasil. Ministério da Saúde. Agência Nacional de Vigilância Sanitária. (2009). Alegações de propriedade funcional aprovadas. Brasília: 2009. Disponível em:<http://portal.anvisa.gov.br/wps/content/Anvisa+Portal/Anvisa/Inicio/Alimentos/Assuntos $+\mathrm{de}+$ Interesse/Alimentos+Com+Alegacoes+de+Propriedades+Funcionais+e+ou+de+Saude/A legacoes+de+propriedade+funcional+Aprovadas>. Acesso em: 03/05/ 2018.

Brasil. Ministério da Saúde. Agência Nacional de Vigilância Sanitária. (2012). Resolução no 54, de 12 de novembro de 2012. Regulamento técnico sobre informação nutricional complementar (declarações de propriedades nutricionais). Diário Oficial da União, Poder Executivo, Brasília, DF.

Brasil. Ministério da Saúde. Agência Nacional de Vigilância Sanitária. (2005). Resolução no 263, de 23 de setembro de 2005. Aprova o Regulamento técnico para produtos de cereais, amidos, farinhas e farelos. Diário Oficial da União, Poder Executivo, Brasília, DF. 
Brasil. Ministério da Saúde. Agência Nacional de Vigilância Sanitária. (2001). Resolução RDC $\mathrm{n}^{\mathrm{o}}$ 12, de 02 de janeiro de 2001. Regulamento Técnico sobre Padrões Microbiológicos para Alimentos. Diário Oficial da União, Poder Executivo, Brasília, DF.

Caetano, B. F. R., Moura, N. A., Almeida, A. P. S., Dias, M. C., Sivieri, K., Barbisan, L. F. (2016). Yacon (Smallanthus sonchifolius) as a food supplement: health-promoting benefits of fructooligosaccharides. Nutrients, 8(7), 436.

Cao, Y., Ma, Z. F., Zhang, H., Jin, Y., Zhang, Y., Hayford, F. (2018). Phytochemical Properties and Nutrigenomic Implications of Yacon as a Potential Source of Prebiotic: Current Evidence and Future Directions. Foods, 7(4), 59.

Cardoso, E. C. (2017). Elaboração de cookies enriquecido com farinha de yacon. (Trabalho de Conclusão de Curso). Universidade Tecnológica Federal do Paraná, Curitiba, Paraná.

Casemiro, I. P., Ramos, P. (2015). Produção científica sobre alimentos funcionais: uma análise das publicações brasileiras entre 2007 e 2013. DEMETRA: Alimentação, Nutrição \& Saúde, 9(4), 925-941.

Castro, A., Caballero, M., Herbas, A., Carballo, S. (2012). Antioxidants in yacon products and effect of long term storage. Food Science and Technology, 32(3), 432-435.

Contado, E. W. N. F., Rocha, D. A., Queiroz, E. R., Abreu, C. M. P. Ramos., E. (2015). Emprego da farinha e do extrato de frutanos de yacon na elaboração de apresuntados. Brazilian Journal of Food Technology, 18(1),49.

Costa, L. L. Estudo reológico, físico-químico e sensorial do uso de farinha de semente de abóbora (Cucurbita sp.) na elaboração de pão de forma. (2014). (Dissertação de Mestrado em Ciência e Tecnologia de Alimentos). Instituto Federal de Educação, Ciência e Tecnologia do Triângulo Mineiro, Uberaba, Minas Gerais.

Delgado, G. T. C., Thomé, R., Gabriel, D. L., Tamashiro, W. M. S. C., Pastore, G. M. (2012). Yacon (Smallanthus sonchifolius) - derived fructooligosaccharides improves the immune parameters in the mouse. Nutrition Research, 32(11), 884-892. 
Delgado, G. T. C., Tamashiro, W. M. S. C., Junior, M. R. M., Pastore, G. M. (2013). Yacon (Smallanthus sonchifolius): a functional food. Plant foods for Human Nutrition, 68(3), 222228.

Goes, T. S., Dionísio, A. P., Modesto, A. L. G., Araújo, I. M. S., Pontes, D. F., Oliveira, R. P., Crisóstomo, C. C. (2014). Elaboração de biscoitos tipo cookie com farinha de resíduos do processamento de extrato de yacon. Blucher Chemical Engineering Proceedings, 1(2), 37413748 .

Habib, N. C., Honoré, S. M., Genta, S. B., Sánchez, S. S. (2011). Hypolipidemic effect of Smallanthus sonchifolius (yacon) roots on diabetic rats: biochemical approach. Chemicobiological interactions, 194(1), 31-39.

Métodos físico-químicos para análise de alimentos (2008). São Paulo, Instituto Adolfo Lutz, $1^{a}$ ed. digital, 1020.

Lobo, A. R., Lobo, A. R., Colli, C., Alvares, E. P., Filisetti, T. M. C. C. (2007). Effects of fructans-containing yacon (Smallanthus sonchifolius Poepp \& Endl.) flour on caecum mucosal morphometry, calcium and magnesium balance, and bone calcium retention in growing rats. British Journal of Nutrition, 97(4), 776-785.

Maldonado, S., Singh, J. C. (2008). Efecto de gelificantes en la formulación de dulce de yacón. Food Science and Technology, 28(2), 429-434.

Malta, D. C., Gosch, C. S., Buss, P., Rocha, D. G., Rezende, R., Freitas, P. C., Akerman, M. (2014). Chronic Non communicable Diseases and the support of intersectorial action to tackle them. Ciência \& Saúde Coletiva, 19(11), 4341-4350.

Malta, D. C., Oliveira, T. P., Santos, M. A. S., Andrade, S. S. C. A., Silva, M. M. A. (2016). Progress with the Strategic Action Plan for Tackling Chronic Non-Communicable Diseases in Brazil, 2011-2015. Epidemiologia nos Serviços de Saúde, 25(2), 373-390. 
Malta, D. C., Bernal, R. T. I., Lima, M. G., Araújo, S. S. C., Silva, M. M. A., Freitas, M. I. F., Barros, M. B. A. (2017). Non communicable diseases and the use of health services: analysis of the National Health Survey in Brazil. Revista de Saúde Pública, 51 (1).

Meilgaard, M., Civille, G. V., Carr, B. T. (2006). Sensory evaluation techniques. (4. Ed). Boca Raton: Taylor \& Francis.

Moraes, C. B., Scheid, M. M. B. (2011). Produção de pães com yacon e avaliação de aceitação pelos diabéticos. XIV Encontro Latino Americano de Iniciação Científica e X Encontro Latino Americano de Pós-Graduação. Universidade do Vale do Paraíba, São José dos Campos, São Paulo.

Oliveira, N. M. A. L., Maciel, J. F., Lima, A. S., Salvino, E. M., Maciel, C. E. P., Oliveira, D. P. M. N., Farias, L. R. G. (2011). Características físico-químicas e sensoriais de pão de forma enriquecido com concentrado protéico de soro de leite e carbonato de cálcio. Revista do Instituto Adolfo Lutz (Impresso), 70(1), 16-22.

Padilha, V. M., Rolim, P. M., Salgado, S. M., Livera, A. S., Andrade, S. A. C., Guerra, N. B.. (2010). Perfil sensorial de bolos de chocolate formulados com farinha de yacon (Smallanthus sonchifolius). Ciência e Tecnologia de Alimentos, 30(3), 735-740.

Pasini, I. S., Doneda, D. (2016). Elaboração e análise sensorial de pães elaborados como alternativa para pacientes com fenilcetonúria. (Trabalho apresentado no Salão de Iniciação Científica XXVIII) Universidade Federal do Rio Grande do Sul, Porto Alegre, RS.

Pereira, J. A. R., Barcelos, M. F. P., Pereira, M. C. A., Ferreira, E. B. (2013). Studies of chemical and enzymatic characteristics of Yacon (Smallanthus sonchifolius) and its flours. Food Science and Technology, Campinas, 33(1), 75-83.

Pereira, A. S. et al. (2018). Metodologia da pesquisa científica. [e-book]. Santa Maria. Ed. UAB/NTE/UFSM. Disponível em: https://repositorio.ufsm.br/bitstream/handle/1/15824/Lic_Computacao_Metodologia-PesquisaCientifica.pdf?sequence=1. 
Rahaie, S., Gharibzahedi, S. M. T., Razavi, S. H., Jafari, S. M. (2014). Recent development son new formulations based on nutrient-dense ingredients for the production of healthyfunctional bread: a review. Journal of Foods science and Technology, 51(11), 2896-2906.

Ribeiro, J. A. (2008). Estudos químico e bioquímico do yacon (Smallanthus sonchifolius) in natura e processado e influência do seu consumo sobre níveis glicêmicos e lipídeos fecais de ratos. (Dissertação de Mestrado em Ciência dos Alimentos). Universidade Federal de Lavras. Lavras, Minas Gerais.

Rodrigues, M. G. G., Santos, E. F., Sanches, F. L. F. Z., Novello, D., Manhani, M. R., Neumann, M. (2014). Desenvolvimento de cookies adicionados de farinha de yacon (Smallanthus sonchifolius): caracterização química e aceitabilidade sensorial entre portadores de Diabetes Mellitus. Revista do Instituto Adolfo Lutz, 73(2), 219-225.

Rolim, P. M. (2008). Aspectos sensoriais e funcionais de pães com farinha de yacon (Smallanthus sonchifolius). (Dissertação de Mestrado em Nutrição). Universidade Federal de Pernambuco. Recife, Pernambuco.

Rolim, P. M., Salgado, S. M., Padilha, V. M., Livera, A. V. S., Andrade, S. A. C., Guerra, N. B. (2011). Glycemic profile and prebiotic potential" in vitro" of bread with yacon (Smallanthus sonchifolius) flour. Food Science and Technology, 31(2), 467-474.

Santana, I., \& Cardoso, M. H. (2008). Yacon tuberous root (Smallanthus sonchifolius): cultivation potentialities, technological and nutritional aspects. Ciência Rural, 38(3), 898-905.

Sant'anna, M. S. L., Rodrigues, V. C., Araújo, T. F., Oliveira, T. T., Peluzio, M. C. G., Ferreira, C. L. L. F (2015). Yacon-Based Product in the Modulation of Intestinal Constipation. Journal of Medicinal Food, 18(9), 980-986.

Silva, N., Junqueira, V. C. A., Silveira, N. F. A., Taniwaki, M. H., Gomes, R. A. R., Okazaki, M. M. (2010). Manual de métodos de análise microbiológica de alimentos. São Paulo, Editora Varela. 
Sivam, A. S., Sun-Waterhouse, D., Waterhouse, G. I. N., Quek, S. Y., Perera, C. O. (2011). Physicochemical properties of bread dough and finished bread with added pectin fiber and phenolic antioxidants. Journal of Food Science, 76(3).

Talma, S. V., Pimentel, C. M., Barbosa, J. B., Pereira, S. M. F. (2011). Avaliação da Qualidade dos Pães para Hamburguer Fabricados em Campos dos Goytacazes-RJ. Biológicas \& Saúde, $1(2)$.

Zaparolli, M. R., Nascimento, N. C., Baptista, D. R., Vayego, S. A. (2013). Alimentos funcionais no manejo da diabetes mellitus. Revista Ciências e Saúde, 6(1), 12-17.

Zavareze, Z. A., Basso, C. (2015). Aceitabilidade de biscoitos de amaranto e yacon. Disciplinarum Scientia Saúde, 16(1), 71-77.

Porcentagem de contribuição de cada autor no manuscrito

Danielly Ramalho de Oliveira - $80 \%$

Ana Paula Colares de Andrade - $20 \%$ 